\title{
Prostatectomía radical: comparación de los resultados obtenidos durante las curvas de aprendizaje de la técnica laparoscópica pura y de la técnica asistida por robot con la prostatectomía radical retropúbica
}

\author{
Caballero Romeu JP*, Palacios Ramos J**, Pereira Arias JG***, Gamarra Quintanilla M**,***, \\ Astobieta Odriozola A**,***, Ibarluzea González G**,***. \\ *Servicio de Urología del Hospital General Universitario de Alicante, Alicante. \\ **Servicio de Urología del Hospital de Galdakao Usansolo, Vizcaya. \\ ***Grupo Urología Clínica, Clínica de la Virgen Blanca, Bilbao, Vizcaya.
}

Actas Urol Esp. 2008;32(10):968-975

\section{RESUMEN}

PROSTATECTOMÍA RADICAL: COMPARACIÓN DE LOS RESULTADOS OBTENIDOS DURANTE LAS CURVAS DE APRENDIZAJE DE LA TÉCNICA LAPAROSCÓPICA PURA Y DE LA TÉCNICA ASISTIDA POR ROBOT CON LA PROSTATECTOMÍA RADICAL RETROPÚBICA

Introducción: La prostatectomía radical retropúbica (PRR) es el patrón oro para el tratamiento quirúrgico del cáncer de próstata organoconfinado. Se están desarrollando técnicas quirúrgicas menos invasivas, como prostatectomía radical laparoscópica (PRL) y la prostatectomía radical asistida por robot (PRAR). El objetivo del estudio es comparar los resultados de la curva de aprendizaje de la PRL y de la PRAR con los de la PRR, especialmente la duración de la intervención quirúrgica.

Material y métodos: Realizamos un estudio observacional retrospectivo Seleccionamos todas las PRR desde Enero de 2000, todas las PRL realizadas en el Hospital de Galdakao y las primeras 60 PRAR realizadas por el Grupo de Urología Clínica. Se evaluaron los parámetros operatorios y perioperatorios y las complicaciones quirúrgicas, comparando posteriormente las tres técnicas.

Resultados: La duración de la intervención fue de $210 \mathrm{~min}$ en la PRR, $345 \mathrm{~min}$ en la PRL y 210 en la PRAR (p $<0,001)$. El sangrado intraoperatorio fue de $1500 \mathrm{ml}$ en la PRR, $1275 \mathrm{ml}$ en la PRL y $400 \mathrm{ml}$ en la PRAR (p < 0,001). A los 6 meses de la intervención la tasa de continencia era del 60\% en el grupo de PRAR, del 45,90\% en el grupo de PRR y del 36,40\% en el grupo de PRL (p = 0,001).

Conclusiones: La PRL requiere un aprendizaje más largo que la PRAR. La PRAR nos ha permitido terminar la intervención en el mismo tiempo que la PRR. En nuestro medio la PRAR demostró ser beneficiosa en términos de estancia postquirúrgica y de sangrado.

Palabras clave: Cáncer de próstata. Laparoscopia. Morbilidad. Prostatectomía radical retropúbica. Incontinencia urinaria. Complicaciones intraoperatorias y sangrado intraoperatorio.

\begin{abstract}
RADICAL PROSTATECTOMY: EVALUATION OF LEARNING CURVE OUTCOMES LAPAROSCOPIC AND ROBOTIC-ASSISTED LAPAROSCOPIC TECHNIQUES WITH RADICAL RETROPUBIC PROSTATECTOMY

Introduction/objective: Radical retropubic prostatectomy (RRP) is the gold standard for the surgical treatment of localized prostate cancer. New techniques are being developed with less invasive methods, including laparoscopic radical prostatectomy (LRP) and robotic-assisted laparoscopic radical prostatectomy (RALP). The aim of the study is to compare LRP and RALP outcomes during the learning curve with RRP, especially operative time and surgical complications.

Material and method: We performed a retrospective observational study of all the RRP cases attended from January 2000 , all the LRPs performed at the Urology Department of the Galdakao Usansolo Hospital and the first 60 RALPs treated by the Clínic Urology group. Baseline parameters, operative and perioperative parameters (nerve preservation, positive margins, intraoperative bleeding, duration of catheterization, hospital stay) and surgical complications were assessed, and the three techniques were compared.

Results: The total number of patients was 192. The mean time operation was of $210 \mathrm{~min}$ in the RRP group, $345 \mathrm{~min}$ in the LRP group and $209.5 \mathrm{~min}$ in the RALP group ( $\mathrm{p}=0$ ). Intraoperative bleeding was of $1500 \mathrm{~mL}$ in RRP, $1275 \mathrm{~mL}$ in LRP and 400 $\mathrm{mL}$ in RALP ( $\mathrm{p}=0$ ) (Table 1 ). Six months after the procedure the continence rate was $60 \%$ in the RALP group, $45.90 \%$ in the RRP group and $36.40 \%$ in the group LRP $(p=0.001)$ (Table 2$)$.

Conclusions: Laparoscopic radical prostatectomy requires a longer learning curve than robotic-assisted prostatectomy. Operative time in RALP procedures was comparable to RRP cases. RALP showed benefits in terms of continence and intraoperative bleeding.
\end{abstract}

Keywords: Prostate cancer. Laparoscopy. Morbidity. Radical retropubic prostatectomy. Urinary incontinence. Intraoperative complications. Blood Loss, Surgical.

Abreviaturas: PRR: Prostatectomía Radical Retropúbica; PRL: Prostatectomía Radical Laparoscópica Pura; PRAR: Prostatectomía Radical Laparoscópica Asistida por Robot. 
$\mathrm{L}_{\mathrm{p}}^{\mathrm{a}}$ a prostatectomía radical retropúbica (PRR) ${ }^{1}$ es el patrón oro en el tratamiento quirúrgico del cáncer de próstata organoconfinado. El auge de las técnicas mínimamente invasivas y la aportación de nuevos conocimientos anatómicos, especialmente en lo que respecta a las bandeletas neurovasculares, ha favorecido el desarrollo de la prostatectomía radical laparoscópica (PRL) desde que en 1997 Schuessler et al. describieron el abordaje laparoscópico y Guilloneau et al., en 1999, sistematizaron la técnica $^{2,3}$. Posteriormente en 2000 Abbou et al. describieron la prostatectomía laparoscópica asistida por robot (PRAR) ${ }^{4}$.

En Europa, y más concretamente en España, se está extendiendo el abordaje laparoscópico y son muy pocos los grupos que disponen del equipo necesario para realizar una PRAR ${ }^{5}$.

Diversos autores han comparado la PRR con la PRL, la PRR con la PRAR, o la PRL con la PRAR. Sin embargo no tenemos conocimiento de que ningún grupo haya comparado sus resultados en las 3 técnicas al mismo tiempo.

Tradicionalmente la curva de aprendizaje se evaluaba exclusivamente por la duración de la intervención. Sin embargo las peculiaridades de la prostatectomía radical obligan a evaluar dicha curva con parámetros no sólo de tiempo quirúrgico sino también según los resultados funcionales y oncológi$\cos ^{6}$. Como otros autores nuestros resultados a nivel oncológico no podrán ser evaluados a corto plazo.

El objetivo de nuestro estudio es comparar los resultados de la curvas de aprendizaje de PRL y PRAR en concepto de tiempo quirúrgico y complicaciones postquirúrgicas con los resultados de la PRR como técnica estándar.

\section{PACIENTES Y MÉTODOS \\ Selección de los pacientes}

Realizamos un estudio observacional retrospectivo de los resultados de la curva de aprendizaje de la PRL y la PRAR en comparación con los resultados de la PRR. Estudiamos a la población intervenida en el Servicio de Urología del Hospital de GaldakaoUsansolo (Vizcaya, España) y por el grupo de "Urología Clínica" de Bilbao (Vizcaya, España).

El criterio de inclusión es cumplir una de las siguientes características: 1) haber sido intervenido mediante PRR desde Enero de 2000 en el Hospital de Galdakao-Usansolo, 2) haber sido intervenido mediante PRL en el Hospital de Galdakao-Usansolo o 3) ser uno de los primeros 60 pacientes intervenidos por el grupo de "Urología Clínica" mediante PRAR. El criterio de exclusión es haber sido intervenido por un cirujano externo a uno de los 3 centros antes mencionados.

Se incluye en el estudio a un total de 192 pacientes: 62 pacientes intervenidos mediante PRR, 70 por PRL y 60 por PRAR. No se incluye en el análisis a 2 pacientes del grupo de las PRAR al ser intervenidos por cirujanos que no pertenecen a los centros mencionados.

\section{Seguimiento y recogida de datos}

La recogida de datos se realiza a partir de las Historias Clínicas de los pacientes teniendo en cuenta los eventos surgidos hasta los 6 meses tras la intervención. Los pacientes son dados de alta según los criterios médicos y teniendo en cuenta la situación y el entorno social del mismo. Los pacientes intervenidos mediante PRR y PRL son dados de alta con sonda vesical y se les cita a los 14-21 días para retirarla sin cistografía previa. A los intervenidos por PRAR se les cita a los 7-14 días para retirar la sonda sin cistografía previa. Los pacientes son valorados el número de veces que su urólogo crea conveniente según la evolución clínica de paciente. Los pacientes son revisados al menos 3 veces dentro de los 6 primeros meses.

Los datos recogidos son: la edad, el ASA, el tacto rectal, el tamaño prostático medido por ecografía transrectal, el PSA preoperatorio, la técnica quirúrgica empleada (PRR, PRL o PRAR), la duración de la intervención (tiempo de anestesia), la conservación o no de bandeletas, el sangrado intraoperatorio, las unidades de concentrado de hematíes transfundidas, la reconversión a cirugía abierta, el Gleason, el estadio tumoral patológico según la clasificación TNM de 1997 y los márgenes quirúrgicos positivos. También se identifica la presencia o no de complicaciones como: fístula urinaria, infección de la herida quirúrgica, dehiscencia de suturas, síndrome miccional, duración del cateterismo vesical, infección del tracto urinario, incontinencia urinaria a los 6 meses de la intervención, el PSA a los 3 meses de la intervención.

Cuando se reconvierte la cirugía se mantiene el análisis según la técnica que se inicia. El sangrado 
intraoperatorio es el que refleja el anestesista en su parte anestésico. El grado Gleason lo dividimos en tres grupos: de Bajo Grado hasta un grado combinado de 6, de Grado Intermedio con un grado combinado de 7 y de Alto Grado con un Gleason combinado mayor de 7 . La incontinencia urinaria la dividimos en 2 grupos: continencia completa o uso de alguna compresa o pañal.

\section{Técnica quirúrgica}

La técnica que se emplea en PRR es la descrita por Walsh ${ }^{7}$. La anastomosis uretrovesical se realiza con de 4 a 6 puntos sueltos de Vycril de 3/0. En la técnica Laparoscópica y en la Robótica se emplea la técnica transperitoneal descrita por el equipo del Instituto Vattikuti ${ }^{8}$ y una sutura tipo Van Velthoven. El nudo de la sutura puede ser sustituido por un Lapra-Ty.

En los 3 grupos se realiza la preservación de las bandeletas neurovasculares siempre siguiendo criterios oncológicos y según la destreza quirúrgica. Se realiza una linfadenectomía ilio-obturatriz bilateral si el $\mathrm{PSA} \geq 10 \mathrm{ng} / \mathrm{ml}$ y/o Gleason combinado $>7$.

La PRR es realizada por 8 miembros diferentes del staff desde hace más de 15 años. Mientras la PRL se realiza por 6 miembros del Servicio de Urología del Hospital de Galdakao Usansolo desde Abril de 2004. Los primeros casos de PRAR tienen lugar desde Enero de 2006 hasta Marzo de 2007 y las intervenciones las realizan 3 cirujanos diferentes.
Los 3 cirujanos que realizan los procedimientos mediante PRAR cuentan con una experiencia limitada en PRL de entre 2 y 10 casos.

\section{Análisis estadístico}

El análisis estadístico se realiza con el programa SPSS 12.0. Para el análisis descriptivo de las variables cuantitativas se utiliza la mediana y percentiles 25 y 75 dado que se verificó con la prueba de Kolmogorov-Smirnov que no seguían una distribución paramétrica. Para el estudio de asociación entre el tipo de intervención y las variables cualitativas (infección urinaria, fístula, etc...) se emplea la prueba de la Chi-cuadrado; y con las variables cuantitativas (días de estancia, etc...) se utiliza la prueba de Kruskall-Wallis. Para ajustar el nivel de significación de la asociación entre la intervención y las complicaciones con aquellas variables que no se distribuyen homogéneamente entre los 3 tipos de cirugía (edad, volumen prostático, PSA, y ASA) se realiza un análisis multivariante del tipo de regresión logística y Análisis de la varianza para más de un factor de variación. El nivel de significación estadística aceptado es $\mathrm{p}<0,05$.

\section{RESULTADOS}

Los datos demográficos de los pacientes aparecen en la Tabla 1. Hay diferencias estadísticamente significativas en cuanto a la edad, el volumen prostático, el PSA y el ASA de los pacientes.

Tabla 1. Características clínicas y patológicas de los pacientes.

\begin{tabular}{|c|c|c|c|c|}
\hline Variable & PRR (n = 62) & PRL (n = 70) & PRAR (n = 60) & $\mathbf{p}$ \\
\hline \multicolumn{5}{|l|}{ Edad } \\
\hline Mediana $\left(\mathrm{P}_{25}-\mathrm{P}_{75}\right)$ & $66,5(62-69)$ & $64(59,5-69)$ & $56(56-65,25)$ & $<0,001$ \\
\hline \multicolumn{5}{|l|}{ Vol. prostático $\left(\mathrm{cm}^{3}\right)$} \\
\hline Mediana $\left(\mathrm{P}_{25}-\mathrm{P}_{75}\right)$ & $41(30,15-52)$ & $34,5(25-49,86)$ & $29,5(23-40)$ & $<0,001$ \\
\hline Tacto rectal & & & & NS \\
\hline No Sospechoso para Ca de Próstata & $24(50 \%)$ & $33(50 \%)$ & $13(38,2 \%)$ & \\
\hline Sospechoso para Ca de Próstata & $24(50 \%)$ & $33(50 \%)$ & $21(61,8 \%)$ & \\
\hline \multicolumn{5}{|l|}{ PSA (ng/dl) } \\
\hline Mediana $\left(\mathrm{P}_{25}-\mathrm{P}_{75}\right)$ & $9.66(7-16.6)$ & $8,69(6,63-11,81)$ & $7(5,7-10)$ & $<0,001$ \\
\hline \multicolumn{5}{|l|}{ ASA n (\%) } \\
\hline I & $5(8,6 \%)$ & $4(5,8 \%)$ & $21(43,8 \%)$ & $<0,001$ \\
\hline II & $46(79,3 \%)$ & $54(78,3 \%)$ & $24(50 \%)$ & \\
\hline III & $7(12,1 \%)$ & $11(15,9 \%)$ & $3(6,3 \%)$ & \\
\hline \multicolumn{5}{|l|}{ Gleason } \\
\hline Bajo & $34(57,6 \%)$ & $37(56,9 \%)$ & $21(36,8 \%)$ & 0,1 \\
\hline Intermedio & $14(23,7 \%)$ & $20(30,8 \%)$ & $22(38,6 \%)$ & \\
\hline Alto & $11(18,6 \%)$ & $8(12,3 \%)$ & $14(24,6 \%)$ & \\
\hline Estadio local patológico (\%) & & & & NS \\
\hline Localizado $\left(\mathrm{pT}_{0}-\mathrm{pT}_{2 \mathrm{c}}\right)$ & 72,4 & 83,8 & 68,4 & \\
\hline Localmente avanzado $\left(\mathrm{pT}_{3 \mathrm{a}}-\mathrm{pT}_{4}\right)$ & 27,6 & 16,2 & 31,6 & \\
\hline
\end{tabular}

p.: nivel de significación estadística 
En la Tabla 2 vemos los resultados obtenidos según cada una de las 3 técnicas. La duración de la PRR (210 min) y de la PRAR (210 min) es significativamente inferior a la de la PRL (345 min) (p.a. $<0,001)$. En el grupo de la PRL hay 22 reconversiones a cirugía abierta por 0 en el grupo de las PRAR.

El porcentaje de pacientes transfundidos en el grupo de PRAR fue de 10,7\% frente al 81,5\% y 48,4\% de la PRR y la PRL respectivamente (p.a. <0,001).

Los márgenes positivos afectaron al 51,9\% de los intervenidos por PRR, al 46.3\% del grupo de PRL y al 30.9\% del grupo de PRAR sin encontrar diferencias estadísticamente significativas.

La estancia postquirúrgica fue menor en el grupo de PRAR, 5 días, por los 8 días de la PRR y la PRL. La duración del cateterismo vesical fue menor en las PRAR 12 días frente a los 22 de la PRR y de la PRL.
En cuanto a las complicaciones quirúrgicas (Tabla 3), la frecuencia de fístula urinaria fue de 1,70\% en las PRAR, de $1.60 \%$ en las PRR y 10,10\% en las PRL sin encontrar diferencias estadísticamente significativas. En lo que respecta a la incontinencia el 60\% de los pacientes intervenidos con robot fueron totalmente continentes a los 6 meses de la intervención frente a $45,90 \%$ en la PRR y a un $36,40 \%$ en la PRL.

Observamos otras complicaciones derivadas de la cirugía (Tabla 4) siendo la más frecuente la fiebre $>38^{\circ} \mathrm{C}$ en 17 casos en el global de la serie, la esclerosis de cuello en 10 casos y el hematoma postquirúrgico en 9. Las complicaciones más graves son la perforación de recto y de ciego que en uno de los casos desencadenó una peritonitis aguda. Hubo 4 casos de tromboembolismo pulmonar 1 en el grupo de la PRR, 1 en el grupo de la PRL y 2 en el grupo de la PRAR.

Tabla 2. Parámetros quirúrgicos y postquirúrgicos.

\begin{tabular}{|c|c|c|c|c|c|}
\hline & PRR (n = 62) & PRL (n = 70) & PRAR $(n=60)$ & $\boldsymbol{p}$ & p.a. \\
\hline \multicolumn{6}{|l|}{ Duración intervención (min) } \\
\hline Mediana $\left(\mathrm{P}_{25}-\mathrm{P}_{75}\right)$ & $210(160-240)$ & $345(315-375)$ & $210(158-240)$ & $<0,001$ & $<0,001$ \\
\hline Reconversiones & - & 22 & - & $<0,001$ & $<0,001$ \\
\hline \multicolumn{6}{|c|}{ Sangrado intraoperatorio $(\mathrm{mL})$} \\
\hline Mediana $\left(\mathrm{P}_{25}-\mathrm{P}_{75}\right)$ & $1500(1200-2000)$ & $1275(700-2000)$ & $400(213-500)$ & $<0,001$ & $<0,001$ \\
\hline Transfusión sanguinea (\%) & $81,50 \%$ & $48,40 \%$ & $10,70 \%$ & $<0,001$ & $<0,001$ \\
\hline \multicolumn{6}{|l|}{ Unidades transfundidas (n) } \\
\hline Mediana $\left(\mathrm{P}_{25}-\mathrm{P}_{75}\right)$ & $2(2-4)$ & $0(0-3)$ & $0(0-0)$ & $<0,001$ & $<0,001$ \\
\hline \multicolumn{6}{|l|}{ Dias de sonda vesical (d) } \\
\hline Mediana $\left(\mathrm{P}_{25}-\mathrm{P}_{75}\right)$ & $22(19-26)$ & $22(17-28)$ & $12(11-14)$ & $<0,001$ & $<0,001$ \\
\hline \multicolumn{6}{|l|}{ Estancia postoperatoria (d) } \\
\hline Mediana $\left(\mathrm{P}_{25}-\mathrm{P}_{75}\right)$ & $8(7-9)$ & $8(5-10)$ & $5(4-6)$ & $<0,001$ & NS \\
\hline Márgenes positivos (\%) & $51,9 \%$ & $46,3 \%$ & $30,9 \%$ & 0,071 & NS \\
\hline
\end{tabular}

p.: nivel de significación estadística

p.a.: nivel de significación estadística ajustado por: edad, volumen prostático, PSA preoperatorio y ASA.

Tabla 3. Complicaciones quirúrgicas.

\begin{tabular}{|c|c|c|c|c|c|c|c|c|}
\hline & \multicolumn{2}{|c|}{ PRR (n = 62) } & \multicolumn{2}{|c|}{ PRL (n = 70) } & \multicolumn{2}{|c|}{ PRAR (n = 60) } & \multirow[t]{2}{*}{$\boldsymbol{p}$} & \multirow[t]{2}{*}{ p.a. } \\
\hline & Porcentaje & Número & Porcentaje & Número & Porcentaje & Número & & \\
\hline ITU & 8,1 & 5 & 5,7 & 4 & 10,3 & 6 & NS & - \\
\hline Infección de la herida & 4,8 & 3 & 7,1 & 5 & 3,4 & 2 & NS & - \\
\hline Dehiscencia sutura cut. & 3,2 & 2 & 5,7 & 4 & 1,7 & 1 & NS & - \\
\hline Fístula urinaria & 1,6 & 1 & 10,1 & 7 & 1,7 & 1 & $<0,05$ & NS \\
\hline Incontinencia§ & 54,1 & 33 & 63,6 & 42 & 40 & 20 & $<0,05$ & NS \\
\hline Síndrome miccional & 16,3 & 8 & 11,5 & 7 & 3,9 & 2 & 0,125 & - \\
\hline
\end{tabular}

p.: nivel de significación estadística

p.a.: nivel de significación estadística ajustado por: edad, volumen prostático, PSA preoperatorio y ASA.

§Incontinencia a 6 meses tras la intervención 
Tabla 4. Otras complicaciones quirúrgicas

\begin{tabular}{lcccc}
\hline Complicaciones perioperatorias & PRR n (\%) & PRL n (\%) & PRAR n (\%) & Total \\
\hline Fiebre $\left(>38^{\circ} \mathrm{C}\right)$ & $8(12,9)$ & $8(11,42)$ & $1(1,66)$ & 17 \\
Esclerosis de cuello vesical & $6(9,67)$ & $3(4,29)$ & $1(1,66)$ & 10 \\
Hematoma & $5(8,06)$ & $1(1,43)$ & $3(5)$ & 9 \\
Daño Rectal & $3(4,84)$ & $4(5,7)$ & 0 & 7 \\
Tromboembolismo & $1(1,61)$ & $1(1,43)$ & $2(2,86)$ & 4 \\
Otras alt. cardiovasculares & $4(6,46)$ & $2(2,86)$ & $1(1,66)$ & 7 \\
Sangrado Postoperatorio & $2(3,23)$ & $1(1,43)$ & 0 & 3 \\
Artralgia & $2(3,23)$ & $1(1,43)$ & 0 & 3 \\
Neumonía & $1(1,61)$ & 0 & $1(1,66)$ & 2 \\
Daño ciego & $1(1,61)$ & 0 & $1(1,66)$ & 2 \\
Peritonitis Aguda & 0 & $1(1,43)$ & $1(1,66)$ & 2 \\
Absceso Abdominal & 0 & $1(1,43)$ & $1(1,66)$ & 2 \\
Litiasis Vesical & $1(1,61)$ & $1(1,43)$ & $1(1,66)$ & 2 \\
Cólico Biliar /Colecistitis & 0 & $2(2,86)$ & 0 & 2 \\
\hline
\end{tabular}

\section{DISCUSION}

En términos de duración de la intervención hemos igualado durante los primeros 58 casos de PRAR los resultados de la PRR. Esto no se cumple en el caso de la PRL. Es difícil comparar los tiempos entre las diferentes series debido a que algunos autores tienen en cuenta el tiempo de "piel a piel" mientras otros tienen en cuenta el tiempo de anestesia. Otros factores como el tamaño prostático, el estadio tumoral, la conservación o no de bandeletas o el tipo de anastomosis uretro-vesical pueden modificar significativamente los tiempos quirúrgicos. Comparado con la PRL, en la PRAR, el mayor número de grados de libertad del robot acorta los tiempos de en la disección, en el posicionamiento de la aguja y en la sutura. El inconveniente es que no tenemos la sensación de tacto y es más difícil controlar la tensión de los hilos. Durante las primeras intervenciones debemos ser cuidadosos para no romper los hilos al anudar o no desgarrar los tejidos. En nuestra experiencia, actualmente tardamos 45 minutos o 1 hora en realizar una anastomosis uretrovesical mediante PRL, con el Robot se realiza en 30 minutos sin dificultad.

En nuestra serie, la mayor tasa de reconversión en la PRL está influida por 4 factores: el número de cirujanos que realizan la técnica, el inicio de la PRAR tras una mínima experiencia en PRL, la planificación del aprendizaje y la baja densidad de casos, especialmente al inicio de la serie. $\mathrm{Al}$ inicio de la curva de aprendizaje en la PRL se establecieron unos criterios de cara a minimizar la repercusión sobre el paciente: dificultades técnicas para continuar la intervención, tiempo quirúrgico excesivo o cuando se presentara una complicación que no se supiera resolver vía laparoscópica. Debemos destacar que el $60 \%$ de las reconversiones se dieron en los primeros 20 casos de PRL. Con la acumulación de experiencia disminuyó drásticamente la tasa de reconversión. Guilloneau presentó un 10\% de reconversiones en sus primeras $70 \mathrm{PRL}^{9}$ Bentas et al. tuvieron un $5 \%$ de reconversiones en sus primeras $40 \mathrm{PRAR}^{10}$.

La mayoría de los grupos coinciden en que con las técnicas mínimamente invasivas el sangrado y la necesidad de transfusión son menores que con la PRR. En nuestro caso existen diferencias durante el periodo de aprendizaje entre la PRL y la PRAR. En un estudio prospectivo y con una distribución aleatoria de los pacientes entre PRL y PRR, Guazzoni et al. observaron un sangrado de 257,3 en la PRL y de $853,3 \mathrm{ml}$ en la PRR ( $<<0,001)$. También fue estadísticamente significativa la menor tasa de transfusión en el grupo de la PRL ${ }^{11}$. Entre la PRR y la PRAR el grupo de Menon encuentra diferencias importantes en el sangrado en su serie inicial 970 cc vs 379 cc respectivamente ${ }^{12}$. Por el contrario Ahlering no encontró diferencias entre ambas técnicas ${ }^{13}$.

Con las técnicas mínimamente invasivas la visualización de los pequeños vasos así como de los planos avasculares permite reducir el sangrado especialmente en la PRAR. El manejo de la presión de insuflación del $\mathrm{CO}_{2}$ permite contener el sangrado venoso regulando la presión de insuflación en pasos clave como la ligadura del complejo venoso dorsal. Además la ventaja de la cirugía robótica es que somos suficientemente hábiles como para emplear la hemostasia con puntos dada la simplicidad de su ejecución.

La duración del cateterismo vesical viene dado por la confianza que el cirujano deposita en la anastomosis. Las técnicas laparoscópicas permiten visualizar 
perfectamente en qué estado queda la anastomosis uretrovesical. El tiempo de permanencia del catéter vesical es menor en los pacientes intervenidos con robot que en la laparoscopia pura porque la reconstrucción uretrovesical es más precisa y también quizás influidos por la observación de una menor incidencia de fístulas urinarias. Así Bhayani y Patel comparan en sus series la PRR con la PRL y la PRAR respectivamente. En ambos casos sistemáticamente se intenta retirar el catéter vesical a partir del $5^{\circ}$ y $7^{\circ}$ día postoperatorio si la cistografía no muestra alteraciones $^{14,15}$. En cambio en las diferentes series el intervalo de cateterismo vesical en la PRL oscila entre los 5,8 días de media de la serie publicada por Guilonneau et al. en 2001 hasta los 14 días de la serie de Bhayani y col de 2003. En la PRAR el tiempo oscila entre 5,9 y 16,7 días ${ }^{10,16}$. Uno de los objetivos de nuestro grupo es reducir el tiempo de cateterismo vesical conforme acumulemos experiencia.

La menor estancia postoperatoria en las PRAR está influida por el medio en que se realiza cada una de las intervenciones. Sin embargo este mismo sesgo ocurre en las series de Estados Unidos o de los centros privados del resto del mundo. También los grupos que han iniciado en nuestro país la cirugía laparoscópica se pueden ver obligados a justificar la viabilidad de los programas acortando las estancias en mayor medida que en los pacientes intervenidos por vía abierta hasta la fecha. Así en la literatura la estancia postoperatoria para la PRL oscila entre 2 y 12,1 días ${ }^{17,18}$. En la PRAR varía desde menos de 2 días de estancia ${ }^{12,14,16,19}$ hasta los 17,1 días ${ }^{10}$. Esta misma variabilidad ocurre en las series de PRR.

Los márgenes positivos de nuestra serie oscilan entre el 51,9\% de las PRR y el 30,9\% de las PRAR. No disponemos de datos suficientes para diferenciar los márgenes positivos extensos de los puntuales. Según la literatura la incidencia de márgenes positivos globalmente varía entre el 5 y el 53\%. Sin embargo esta incidencia no depende sólo de la experiencia del cirujano sino también del método de análisis anatomopatológico, de la conservación de bandeletas neurovasculares y del estadio y volumen tumoral $^{20}$. Guillonneau publica en 2005 una revisión de los resultados en la PRL de algunos de los centros más importantes del mundo. En estos la tasa de márgenes positivos varía desde un 11,4\% hasta un $26,4 \%{ }^{21}$. Atug et al. analizaron los márge- nes quirúrgicos positivos de sus 100 primeros casos de PRAR divididos en grupos de 33 casos. Los autores hallaron diferencias estadísticamente significativas entre el primer y el tercer grupo concluyendo que al acumular más experiencia se logra una disección meticulosa del ápex y del cuello vesical, fundamental en la reducción de los márgenes positivos ${ }^{22}$.

En cuanto a las complicaciones quirúrgicas, nos llama la atención que no haya diferencias estadísticamente significativas en términos de infección de la herida quirúrgica, dehiscencia de suturas o ITU. Esperábamos una diferencia significativamente menor en los pacientes tratados con PRAR o PRL dado que las incisiones son de menor tamaño y presentan una cicatrización más rápida. La PRL y la PRAR condicionan un importante desgaste del cirujano al final de la intervención, especialmente durante la curva de aprendizaje. Debemos prestar mucha atención al cierre de la laparotomía para beneficiarnos de las ventajas de una herida menor que en la PRR.

El tromboembolismo pulmonar (TEP) es una de las causas más importantes de mortalidad en el postoperatorio de la prostatectomía radical, un $0,5 \%$ de los pacientes. Su incidencia ha llevado a diferentes autores a plantearse una profilaxis del TEP con anticoagulantes. Un estudio multicéntrico coordinado por Secin y que incluye a 5951 pacientes concluye que la profilaxis sistemática no reduce de manera significativa la incidencia de $\mathrm{TEP}^{23}$.

Es muy importante que haya una tendencia en términos de fistulas urinarias y de continencia a los 6 meses a favor de la PRAR en nuestra serie. La continencia afecta mucho a la calidad de vida de los pacientes. Como muchos autores decidimos ser muy exigentes con la definición de "Continencia". Es complicado comparar los resultados de las diferentes series por las diferencias de parámetros como el tipo de disección o el estadio patológico. Queda por dilucidar cómo considerar a aquel paciente que lleva una compresa "de seguridad" o que mancha "unas gotas" al día y su significación clínica para valorar el éxito o el fracaso de la cirugía en este apartado. Tampoco existe un consenso acerca del momento idóneo para establecer la continencia o no del paciente. Esta variabilidad en la evaluación de la incontinencia queda patente en las grandes diferencias entre las series publicadas. En la PRAR esta oscila entre el $4 \%$ y el $40 \%{ }^{10,24}$. En la revisión de los 
resultados en PRL realizada por Guillonneau de 2005 observó una tasa de incontinencia variable desde el 15\% al 0\% según los autores a diferentes intervalos de tiempo ${ }^{21}$.

En nuestra serie el número total de complicaciones fue menor en las técnicas mínimamente invasivas. La complicación más frecuente fue la fiebre durante el postoperatorio. Destaca el mayor número de esclerosis de cuello en los pacientes intervenidos por vía abierta. El tratamiento fue endoscópico y satisfactorio hasta la fecha en todos lo casos. Con el robot no hubo ningún caso de daño rectal mientras que hubo 3 por vía abierta y 4 por vía laparoscópica. Todos se resolvieron durante la intervención con cierre primario con puntos de seda excepto en un caso del grupo de PRL que presentó una peritonitis aguda y requirió una colostomía de descarga. En el grupo de PRAR 2 pacientes presentaron un hematoma importante, uno requirió una laparotomía urgente y ligadura de los vasos sangrantes y al otro se le drenó por vía perineal. En este mismo grupo hubo una perforación de ciego que requirió una cecostomía al $5^{\circ}$ día postoperatorio. También se reintervino por vía abierta a un paciente por arrancamiento de la sonda al $5^{\circ}$ día con el fin de recolocar la sonda y reconstruir la anastomosis. En el grupo de la PRL.

La falta de homogeneidad de los grupos se debe a la lógica selección de casos para desarrollar la curva de aprendizaje en las técnicas mínimamente invasivas. Tratamos de escoger a pacientes jóvenes, con un riesgo anestésico (ASA) bajo y con un volumen prostático intermedio. Esta falta de homogeneidad se ha corregido realizando un análisis multivariante por aquellos parámetros de base con diferencias estadísticamente significativas entre los 3 grupos. El análisis de los datos por la técnica que se inició nos permite también no omitir las peculiaridades de los resultados obtenidos por una técnica en desarrollo.

\section{CONCLUSIONES}

Las técnicas de cirugía mínimamente invasiva deben ser desarrolladas a medida que la capacidad tecnológica y económica lo vaya permitiendo. A priori la PRL y sobretodo la PRAR ofrecen importantes ventajas en cuanto al descenso de la morbimortalidad (sangrado, cateterismo vesical, continencia). Sin embargo estos beneficios deben contrastarse con otros estudios de coste-efectividad. Debería investigarse si la diferencia en los resultados es tan espectacular cuando el incentivo del equipo que desarrolla las diferentes técnicas es el mismo.

A nuestro juicio la cirugía abierta sigue siendo el patrón oro de la cirugía del cáncer de próstata organoconfinado pero las facilidades que ofrece la PRAR en cuanto a aprendizaje y aplicación hace que en el futuro se establezca esta como referencia y sea demandada activamente por los pacientes.

\section{Agradecimientos}

Agradezco a los diferentes urólogos del Hospital de Galdakao-Usansolo y del grupo de Urología Clínica el acceso completo y sin restricciones a las historias clínicas así como la libertad que me han otorgado en la publicación de sus resultados, especialmente a los Dres. José Antonio Gallego, Nemesio Prieto Ugidos y a la Dra. Isabel Camargo que por el límite establecido de autores no pueden aparecer como tal. Gracias también al Dr. Sánchez del Servicio de Medicina Preventiva del Hospital General de Alicante por su apoyo fundamental en el análisis estadístico de los datos.

Han colaborado en la realización de este estudio la Fundación para la Investigación en Urología de la Asociación Española de Urología y la Fundación de la Comunidad Valenciana para la Investigación en el Hospital General Universitario de Alicante.

\section{REFERENCIAS}

1. Walsh PC. Radical Prostatectomy for localized prostate cancer provides durable cancer control with excellent quality of life: a structures debate. J Urol. 2000;163(6):1802-1807.

2. Schuessler WW, Schulman PG, Clayman RV, Kavoussi LR. Laparoscopic radical prostatectomy: initial short term experience. Urology. 1997;50(6):854-857.

3. Guilloneau B, Cathelineau X, Barret E, Rozet F, Vallancien G. Laparoscopic radical prostatectomy: technical and early oncological assesment of 40 operations. Eur Urol. 1999;36(1):14-20.

4. Abbou CC, Hoznek A, Salomon L, Lobontiu A, Saint F, Cicco A et al. Remote laparoscopic radical prostatectomy carried out with a robot. Report of a case. Prog Urol. 2000;10(4):520-523.

5. Mavrich Villavicencio H, Esquena S, Palou Redorta J, Gómez Ruíz JJ.. Prostatectomía radical robótica: revisión de nuestra curva de aprendizaje. Actas Urol Esp. 2007;31(6):587-592.

6. Bollens R, Sandhu S, Roumeguere T, Buackels T, Schulman C. Laparoscopic radical prostatectomy: the learning curve. Curr Opin Urol. 2005;15(2):79-82.

7. Walsh PC. Radical retropubic prostatectomy. In: Walsh PC, Retik $\mathrm{AB}$, Stamey TA, 8. Vaughan ED, eds. Campbells Textbook of Urology. Philadelphia: W.B. Saunders; 1992; 3: 2865-2886.

8. Guilloneau B, Cathelineau X, Doublet JD, Vallancien G. Laparoscopic Radical Prostatectomy: The Lessons Learned. J Endourol. 2001,17(4):441-445. 
9. Bentas W, Wolfram M, Jones J, Bräutigam R, Kramer W, Binder J. Robotic technology and the translation of open radical prostatectomy to laparoscopy: the early Frankfurt experience with robotic radical prostatectomy and one year follow-up. Eur Urol. 2003,44(2):175-181.

10. Guazzoni G, Cestari A, Naspro R, Riva M, Centemero A, Zanoni $\mathrm{M}$, et al. Intra- and perioperative outcomes comparing radical retropubic and laparoscopic radical prostatectomy: results from a prospective, randomized, single-surgeon study. Eur Urol. 2006;50(1):98-104.

11. Menon M, Tewari A, Baize B, Guillonneau B, Vallancien G. Prospctive comparison of radical retropubic prostatectomy and robot-assisted anatomic prostatectomy: the Vattikuti urology institute experience. Urology. 2002,60(5):864-868.

12. Ahlering TE, Woo D, Eichel L, Lee DI, Edwards R, Skarecky DW. Robot-assisted versus open radical prostatectomy: a comparison of one surgeon's outcomes. Urology. 2004;63(5):819-822.

13. Patel VR, Tully AS, Holmes R, Lindsay J. Robotic radical prostatectomy in the community setting-the learning curve and beyond: initial 200 cases. J Urol. 2005,174(1):269-272.

14. Bhayani SB, Pavlovich CP, Hsu TS, Sullivan W, Su LM. Prospective comparison of short-term convalescence: laparoscopic radical prostatectomy versus open radical retropubic prostatectomy. Urology. 2003;61(3):612-616.

15. Zorn KC, Gofrit ON, Orvieto MA, Mikhail AA, Zagaja GP, Shalhav AL. Robotic-Assisted Laparoscopic Prostatectomy: Functional and Pathologic Outcomes with Interfascial Nerve Preservation. Eur Urol. 2007;51(3):755-763.

16. Ghavamian R, Knoll A, Boczo J, Melman A. Comparison of operative and functional outcomes of laparoscopic radical prostatectomy and radical retropubic prostatectomy: single surgeon experience. Urology. 2006;67(6):1241-1246.
17. Poulakis V, Dillenburg W, Moeckel M, de Vries R, Witzsch U, Zumbé J et al. Laparoscopic Radical Prostatectomy: Prospective Evaluation of the Learning Curve. Eur Urol 2005;47(2):167-175.

18. Bhandari A, McIntire L, Kaul SA, Hemal AK, Peabody JO, Menon M. Perioperative complications of robotic radical prostatectomy after the learning curve J Urol. 2005;174(3):915-918.

19. Descazeaud A, Zerbib M, Peyromaure M. Risk factors for positive surgical margins following radical prostatectomy: review. Ann Urol (Paris). 2006;40(6):342-348.

20. Trabulsi EJ, Guillonneau B. Laparoscopic radical prostatectomy. J Urol. 2005;173(4):1072-1079.

21. Atug F, Castle EP, Srivastav SK, Burgess SV, Thomas R, Davis R. Positive surgical margins in robotic-assisted radical prostatectomy: impact of learning curve on oncologic outcomes. Eur Urol. 2006;49(5):866-872.

22. Secin FP, Jiborn T, Bjartell AS, Fournier G, Salomon L, Abbou $\mathrm{CC}$, et al. Multi-institutional study of symptomatic deep venous thrombosis and pulmonary embolism in prostate cancer patients undergoing laparoscopic or robot-assisted laparoscopic radical prostatectomy. Eur Urol. 2008,(53) 134-145.

Correspondencia autor: Dr. J.P. Caballero Romeu Servicio de Urología

Hospital General Universitario de Alicante

Pintor Baeza, s/n - 03010 Alicante

Tel.: 965938310

E-mail autor: juanpablocaballero@gmail.com

Información artículo: Original - Cáncer de próstata

Trabajo recibido: junio 2008

Trabajo aceptado: julio 2008 